\title{
Bread making technology influences postprandial glucose response: a review of the clinical evidence
}

\author{
Nikoleta S. Stamataki, Amalia E. Yanni and Vaios T. Karathanos* \\ Laboratory of Chemistry-Biochemistry-Physical Chemistry of Foods, Department of Nutrition and Dietetics, Harokopio \\ University, 17671 Athens, Greece \\ (Submitted 19 September 2016 - Final revision received 1 March 2017 - Accepted 7 March 2017-First published online 2 May 2017)
}

\begin{abstract}
Lowering postprandial glucose and insulin responses may have significant beneficial implications for prevention and treatment of metabolic disorders. Bread is a staple food consumed worldwide in a daily basis, and the use of different baking technologies may modify the glucose and insulin response. The aim of this review was to critically record the human studies examining the application of different bread making processes on postprandial glucose and insulin response to bread. Literature is rich of results which show that the use of sourdough fermentation instead of leavening with Saccharomyces cerevisiae is able to modulate glucose response to bread, whereas evidence regarding its efficacy on lowering postprandial insulin response is less clear. The presence of organic acids is possibly involved, but the exact mechanism of action is still to be confirmed. The reviewed data also revealed that the alteration of other processing conditions (method of cooking, proofing period, partial baking freezing technology) can effectively decrease postprandial glucose response to bread, by influencing physical structure and retrogradation of starch. The development of healthier bread products that benefit postprandial metabolic responses is crucial and suggested baking conditions can be used by the bread industry for the promotion of public health.
\end{abstract}

Key words: Glycaemic index: Insulinaemic index: Insulin response: Leavening techniques: Sourdough fermentation: Bread processing: Bread volume

Carbohydrates represent the most important dietary energy source for humans quantitatively, and the main determinant of postprandial blood glucose response ${ }^{(1)}$, as the ingestion of carbohydrates is accompanied by an increase in blood glucose levels. On the basis of their effect on postprandial glycaemia, carbohydrate-rich foods are classified into three categories of glycaemic index (GI) (low GI $\leq 55$, medium GI 55-69 or high GI $\geq 70)^{(2)}$. GI indicates the extent to which a carbohydrate containing food affects postprandial blood glucose levels compared with a reference food (glucose or white bread) which contains the same amount of available carbohydrate ${ }^{(3)}$. In the same context, insulinaemic index (II) is a marker that indicates the elevation of blood insulin levels in a 2-h postprandial period. The ingestion of high GI foods cause a high increase in postprandial blood glucose concentrations, whereas the increase is less pronounced for foods with a low GI. A high postprandial blood glucose response, in turn, triggers a disproportionately high insulin response which is associated with the development of hyperinsulinaemia and insulin resistance ${ }^{(4)}$.

Among carbohydrate rich-foods, bread is a staple food consumed in all parts of the world in different forms originating from variations in the ingredients traditionally used and processing techniques applied ${ }^{(5)}$. Even in the same country, bread baking is in continuous development and various methods of baking and different kinds of bread are available. As a consequence, bread's GI values can vary a lot and bread can fall in the category of either low, medium or high $\mathrm{GI}^{(6)}$. Lowering the GI of bread is of scientific interest especially for populations who consume large amounts of bread, such as the Nordic European countries ${ }^{(7)}$. Attenuation of GI can be achieved by different approaches, including the addition of intact structures not accessible to human amylases, viscous and non-viscous fibre ${ }^{(8)}$, legume flours, fruitbased ingredients and enrichment with specific micronutrients ${ }^{(9)}$.

The aim of this review was to examine another significant determinant of glucose response to bread, namely bread making procedures. In particular, the influence of leavening techniques and baking method of bread on postprandial glucose and insulin responses are discussed in this article. Insulin response was also examined in order to get a wider picture of the postprandial metabolic effects caused by processing conditions. Pubmed, Scopus, Sciencedirect and Springer databases were searched for interventional studies regarding GI and/or acute postprandial glucose response to bread using relevant indexing terms. Cited references of

Abbreviations: GI, glycaemic index; II, insulinaemic index; RS, resistant starch; T2D, type 2 diabetes.

* Corresponding author: V. T. Karathanos, fax +30210957 7050, email vkarath@hua.gr 
identified articles were also revised for appropriate missed studies. To include a study in this review, human subjects should have acutely consumed different types of breads, differing in leavening techniques, or other processing parameters, compared with a reference food which was either white wheat bread or glucose, after overnight fast. There were no restrictions in terms of participants' characteristics. Dates of publication were limited to 2000 onward, in an attempt to present updated literature, and only human studies in English language papers were eligible. Identified studies were classified in two categories according to whether breads differed in leavening technique or other processing conditions, and are summarised in Tables 1 and 3, respectively.

\section{Leavening techniques}

\section{Sourdough fermentation}

Sourdough has traditionally been used as leavening agent in bread making, whereas nowadays only few bakeries work with sourdough at an industrial scale. Its use in bread making has a great impact on bread characteristics, including texture, flavour, shelf life and nutritional quality ${ }^{(10)}$. This fermentation technique has primarily been used for wheat and rye baking. Wheat and rye sourdoughs do not exhibit characteristic differences in fermentation microbiota or their metabolic activity ${ }^{(11)}$. Table 1 summarises human trials investigating the effect of sourdough fermentation on postprandial glucose and insulin response to bread, and Table 2 presents selected properties of sourdough breads.

Sourdough fermentation has been proved an effective strategy for lowering the metabolic response to bread due to the production of organic acids ${ }^{(6)}$, whereas the exact mechanisms have not been fully elucidated. Breads enriched with organic acids, either physiologically produced during fermentation or even artificially added, have been shown to improve postprandial glucose and insulin responses in healthy subjects ${ }^{(12)}$. A large number of studies have confirmed the above results not only in healthy individuals ${ }^{(13-15)}$ but also in subjects with impaired glucose tolerance ${ }^{(16-17)}$. Rye sourdough fermented breads have exhibited lower acute postprandial insulin responses ${ }^{(18-19)}$ and occasionally improved glycaemic profiles ${ }^{(18,20)}$.

A sourdough (Lactobacillus plantarum P1 and Lactobacillus brevis P2) wholemeal bread enriched with oat fibre $(3.9 \mathrm{~g}$ $\beta$-glucan $/ 50 \mathrm{~g}$ of available carbohydrates) was found to have low GI (53.7) compared with glucose ${ }^{(15)}$, and a wheat sourdough bread enriched with oat and rye fibre (total dietary fibre content $8 \cdot 13 \mathrm{~g} / 100 \mathrm{~g}$ bread) was found to have GI 41 ( $v$. GI 100 of glucose) in another study ${ }^{(13)}$. The clear effect of sourdough fermentation on the GI was revealed when same breads, which differed only in the leavening technique, were compared ${ }^{(21)}$. Postprandial glucose response was reduced in both sourdough white bread and sourdough wholemeal bread in comparison to breads made with Saccharomyces cerevisiae.

Juntunen et al. ${ }^{(19)}$ studied the acute effect of three rye breads prepared with sourdough fermentation on glucose and insulin responses in comparison with refined wheat bread. The test breads were endosperm rye bread (100\% commercial endosperm rye flour, sourdough), traditional rye bread (100\% wholemeal rye flour, sourdough) and high-fibre rye bread (60\% wholemeal rye flour, $40 \%$ rye bran and sourdough). Sourdough was prepared from either commercial endosperm flour for the endosperm rye bread or wholemeal rye flour for the other two breads, along with L. brevis and L. plantarum, fresh yeast and water. Postprandial AUC for glucose did not significantly differ between the rye breads and refined wheat bread, whereas postprandial insulin AUC was significantly decreased after the ingestion of the endosperm rye bread and the traditional one. The present study showed that in healthy subjects less insulin is needed for the control of glucose levels after the consumption of sourdough rye breads compared with refined wheat bread. However, the lower insulin response cannot be explained by the fibre content, as an insignificant decrease in insulin response caused by the high fibre rye bread ( $29 \mathrm{~g}$ total dietary fibre/50 $\mathrm{g}$ of available carbohydrates). In addition, sourdough fermented endosperm rye bread was also shown to induce lower postprandial insulin response compared with white wheat bread without improving blood glucose profile in healthy subjects in the study by Bondia-Pons et al. ${ }^{(20)}$. In another study, a rye wheat sourdough bread was found to have medium GI $(\mathrm{GI}=62)$ and caused lower postprandial insulin response compared with a soft pretzel ${ }^{(7)}$.

The postprandial metabolic response of sourdough fermented wheat bread was tested in overweight and obese subjects, who represent a target group with increased risk for developing type 2 diabetes (T2D) ${ }^{(14)}$. Glucose AUC (180 min) for sourdough bread (100\% white wheat flour, 37\% sourdough starter from white flour) was significantly lower than for whole wheat bread (71\% whole wheat flour, 29\% whole grain flour), whereas insulin response did not differ between the test breads. The more modest glucose profile without differences in insulin response, may indicate that sourdough increased insulin sensitivity in these individuals. In another study by Lappi et $a l^{(16)}$ the ingestion of sourdough fermented wholemeal wheat bread (100\% wheat flour from peeled grains) by subjects with the metabolic syndrome resulted in retarded postprandial glucose response, and significantly lower maximum insulin increase compared with white bread. AUC for insulin did not differ between the test breads, but a trend for lower AUC after the ingestion of the wholemeal wheat bread produced with sourdough than that of white bread was observed.

The effect of acute postprandial glucose and insulin response to breads varying in carbohydrate quality due to sprouted grains and sourdough fermentation in adult patients with T2D was examined in a study, which showed that breads made of sprouted grains and produced with sourdough fermentation exhibit lower blood glucose incremental AUC (iAUC), compared with whole grain or white wheat sourdough fermented breads $^{(22)}$. The particular type of bread also caused significantly lower insulin response but only in the second meal. The sprouting treatment of cereal grains causes changes in the grain structure and composition. It is reported to decrease starch content and increase the content and availability of vitamins, minerals, and antioxidants. Acute consumption of sprouted-grain sourdough bread had previously shown to improve glycaemia by lowering glucose response in overweight or obese males ${ }^{(23)}$. In this randomised single-blind cross-over design trial, overweight or obese subjects consumed the following bread 


\section{N British Journal of Nutrition}

Table 1. Human studies examining the effect of sourdough fermentation on postprandial glucose $(G)$ and insulin response to bread (Mean values with their standard errors; mean values and standard deviations)

\begin{tabular}{|c|c|c|c|c|c|c|c|}
\hline \multirow[b]{2}{*}{ References } & \multirow[b]{2}{*}{ Bread formulation } & \multirow[b]{2}{*}{ Subjects† } & \multirow{2}{*}{$\begin{array}{l}\text { Total dietary } \\
\text { fibre contentł }\end{array}$} & \multicolumn{2}{|c|}{ Glucose response§ } & \multicolumn{2}{|c|}{ Insulin response§ } \\
\hline & & & & iAUC & Gl & iAUC & II \\
\hline \multirow[t]{2}{*}{ Goletzke et al. ${ }^{(7)}$} & $\begin{array}{l}\text { Wholemeal rye bread with intact grains and } \\
\text { sunflower seeds }(12 \%)\end{array}$ & 12 & 8.0 & $\begin{array}{l}\operatorname{iAUC}(\mathrm{mmol} / / \times \mathrm{min})(120 \mathrm{~min}) \\
108(\mathrm{SEM} 13)\left({ }^{*} v . \mathrm{G}\right)\end{array}$ & 55 (SEM 4) $\left({ }^{*} v \cdot G\right)$ & $\begin{array}{l}\text { iAUC }(\text { pmol/ } / 1 \times \min )(120 \mathrm{~min}) \\
6730(\text { SEM } 1360)\end{array}$ & $70(\operatorname{sem} 10)\left({ }^{*} v \cdot G\right)$ \\
\hline & $\begin{array}{l}\text { Wholemeal spelt wheat bread } \\
\text { Rye wheat sourdough bread } \\
\text { Soft pretzel }\end{array}$ & & $\begin{array}{l}\text { Not provided } \\
6 \cdot 0 \\
\text { Not provided }\end{array}$ & $\begin{array}{l}128\left(\operatorname{SEM~17)}\left({ }^{*} v \cdot \mathrm{G}\right)\right. \\
117\left(\operatorname{SEM~12)}\left(^{*} v \cdot \mathrm{G}\right)\right. \\
169(\operatorname{SEM} 27)\left(^{*} v \cdot \mathrm{G}\right)\end{array}$ & $\begin{array}{l}63\left(\text { SEM 4) }\left({ }^{*} v \cdot G\right)\right. \\
62\left(\text { SEM 4) }\left({ }^{*} v \cdot \mathrm{G}\right)\right. \\
80\left(\text { SEM 6) }\left({ }^{*} v \cdot \mathrm{G}\right)\right.\end{array}$ & $\begin{array}{l}8275(\text { SEM } 2305) \\
6360(\text { (SEM 940) } \\
9220(\text { SEM } 1245)\end{array}$ & $\begin{array}{l}77(\text { SEM } 10)\left({ }^{*} \text { v. G) }\right. \\
72(\text { SEM } 9)\left({ }^{*} v \cdot G\right) \\
102(\text { SEM } 10)\end{array}$ \\
\hline \multirow[t]{4}{*}{ Tucker et al. ${ }^{(22)}$} & $\begin{array}{l}\text { Three-grain sprouted sourdough bread } \\
\text { (whole-grain wheat, rye sourdough and } \\
\text { rolled oats) }\end{array}$ & 12 patients with T2D & $12.5 \mathrm{~g} / 50 \mathrm{~g} \mathrm{AC}$ & \multicolumn{2}{|l|}{$\begin{array}{l}\text { net iAUC }(\mathrm{mmol} / / \times \mathrm{min})(180 \mathrm{~min}) \\
300 \cdot 9 \text { ( } \mathrm{(EM} 35 \cdot 21)\left({ }^{\star} v \text {. all other }\right. \\
\text { breads) }\end{array}$} & \multicolumn{2}{|c|}{$\begin{array}{c}\text { net iAUC }(\mathrm{pmol} / / \times \mathrm{min})(180 \mathrm{~min}) \\
21347(\mathrm{SEM} 4131)\end{array}$} \\
\hline & $\begin{array}{l}\text { Whole-grain wheat (predominately), rye } \\
\text { sourdough bread }\end{array}$ & & $12.0 \mathrm{~g} / 50 \mathrm{~g} \mathrm{AC}$ & \multicolumn{2}{|l|}{369.7 (SEM 42.66) } & \multicolumn{2}{|c|}{24076 (SEM 5234) } \\
\hline & $\begin{array}{l}\text { White flour bread enriched with lactic acid, } \\
\text { ascorbic acid, sodium acetate }\end{array}$ & & $2.02 \mathrm{~g} / 50 \mathrm{~g} \mathrm{AC}$ & \multicolumn{2}{|l|}{378.0 (SEM 45.62) } & \multicolumn{2}{|c|}{20231 (SEM 4482) } \\
\hline & White bread & & $2.64 \mathrm{~g} / 50 \mathrm{~g} \mathrm{AC}$ & $362 \cdot 1$ (SEM 39.0) & & 21938 (SEM & \\
\hline \multirow[t]{2}{*}{ Mofidi et al. ${ }^{(23)} \|$} & $\begin{array}{l}\text { 11-grain (whole grain with sourdough) } \\
\text { Sprouted grain (whole grain with sourdough) } \\
\text { Sourdough WB } \\
\text { 12-grain (whole grain) } \\
\text { White bread }\end{array}$ & 12 overweight or obese & $\begin{array}{l}12.8 \mathrm{~g} / 50 \mathrm{~g} \mathrm{AC} \\
12.1 \mathrm{~g} / 50 \mathrm{~g} \mathrm{AC} \\
5.2 \mathrm{~g} / 50 \mathrm{~g} \mathrm{AC} \\
11.0 \mathrm{~g} / 50 \mathrm{~g} \mathrm{AC} \\
4.9 \mathrm{~g} / 50 \mathrm{~g} \mathrm{AC}\end{array}$ & \multicolumn{2}{|l|}{$\begin{array}{l}\text { IAUC (mmol/l/ } \times \text { min) }(180 \mathrm{~min}) \\
0.64(\text { SEM } 0.04) \\
0.22(\text { SEM } 0.17)\left({ }^{*} v . \text { WB }\right) \\
0.66 \text { (SEM } 0.16) \\
0.26 \text { (SEM } 0.11) \\
0.51(\text { SEM } 0.17)\end{array}$} & \multicolumn{2}{|c|}{ 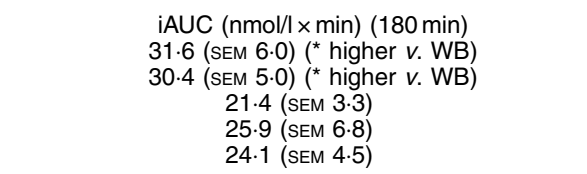 } \\
\hline & $\begin{array}{l}\text { 11-grain (whole grain with sourdough) } \\
\text { Sprouted grain (whole grain with sourdough) } \\
\text { Sourdough WB } \\
12 \text {-grain (whole grain) } \\
\text { White bread }\end{array}$ & 11 overweight or obese & $\begin{array}{l}9.1 \mathrm{~g} / 107 \mathrm{~g} \text { bread } \\
8.2 \mathrm{~g} / 107 \mathrm{~g} \text { bread } \\
5.2 \mathrm{~g} / 107 \mathrm{~g} \text { bread } \\
9.6 \mathrm{~g} / 107 \mathrm{~g} \text { bread } \\
4.8 \mathrm{~g} / 107 \mathrm{~g} \text { bread }\end{array}$ & \multicolumn{2}{|l|}{$\begin{array}{l}0.31 \text { (SEM } 0.12 \text { ) } \\
0.17 \text { (SEM } 0.15) \\
0.72 \text { (SEM } 0.19) \text { (* higher } v . \text { WB) } \\
0.41 \text { (SEM } 0.11) \\
0.46 \text { (SEM } 0.14 \text { ) }\end{array}$} & $\begin{array}{r}16 \cdot 2 \text { (SEM } \\
12.7 \text { (SEM } \\
21.5 \text { (SEM } \\
16 \cdot 8 \text { (SEM } 2 \cdot 4) \\
18 \cdot 1 \text { (SEM }\end{array}$ & f. WB) \\
\hline \multirow[t]{4}{*}{ Novotni et al. ${ }^{(32)}$} & $\begin{array}{l}\text { Control gluten-free partial baked frozen } \\
\text { bread without sourdough }\end{array}$ & \multirow[t]{4}{*}{11} & $7.8 \mathrm{~g} / 50 \mathrm{~g} \mathrm{AC}$ & & 68 (SEM 7) & & \\
\hline & $\begin{array}{l}7.5 \mathrm{~g} \text { sourdough } / 100 \mathrm{~g} \text { bread batter } \\
15 \mathrm{~g} \text { sourdough } / 100 \mathrm{~g} \text { bread batter }\end{array}$ & & $\begin{array}{l}7.6 \mathrm{~g} / 50 \mathrm{~g} \mathrm{AC} \\
8.0 \mathrm{~g} / 50 \mathrm{~g} \mathrm{AC}\end{array}$ & & $\begin{array}{l}59(\text { SEM } 6) \\
52 \text { (SEM } 3)\left(^{*} v . \text { control }\right. \\
P=0.07)\end{array}$ & & \\
\hline & $22.5 \mathrm{~g}$ sourdough $/ 100 \mathrm{~g}$ bread batter & & $8.1 \mathrm{~g} / 50 \mathrm{~g} \mathrm{AC}$ & & $54($ SEM 6$))^{*}{ }^{*}$ v. control & & \\
\hline & $30 \mathrm{~g}$ sourdough $/ 100 \mathrm{~g}$ bread batter & & $7.6 \mathrm{~g} / 50 \mathrm{~g} \mathrm{AC}$ & & 61 (SEM 6) & & \\
\hline \multirow[t]{2}{*}{ Novotni et al. ${ }^{(33)}$} & $\begin{array}{l}\text { Control wholemeal partially frozen baked } \\
\text { bread (without sourdough) } \\
10 \% \text { sourdough wholemeal wheat partially } \\
\text { frozen baked breads: }\end{array}$ & \multirow[t]{2}{*}{10} & $8.4 \mathrm{~g} / 50 \mathrm{~g} \mathrm{AC}$ & & 70 (SEM 7.1) & & \\
\hline & $\begin{array}{l}\text { Lactobacillus plantarum } \\
\text { Lactobacillus fermentum (PL1) } \\
\text { L. fermentum with phytase (PL3) } \\
\text { Lactobacillus brevis combined with } \\
\text { Saccharomyces cerevisiae var. chevalieri } \\
\text { (LV4) }\end{array}$ & & $\begin{array}{l}8.0 \mathrm{~g} / 50 \mathrm{~g} \mathrm{AC} \\
8.5 \mathrm{~g} / 50 \mathrm{~g} \mathrm{AC} \\
8.3 \mathrm{~g} / 50 \mathrm{~g} \mathrm{AC} \\
8.2 \mathrm{~g} / 50 \mathrm{~g} \mathrm{AC}\end{array}$ & & $\begin{array}{l}60 \text { (SEM 6.5) } \\
56 \text { (SEM 6.4) (* v. control) } \\
56 \text { (SEM 5.5) ( }\left(^{*} \text { v. control) }\right. \\
50 \text { (SEM 4.6) (** v. control) }\end{array}$ & & \\
\hline \multirow[t]{2}{*}{$\begin{array}{l}\text { Bondia-Pons } \\
\text { et al. }\end{array}$} & $\begin{array}{l}\text { Commercial sourdough endosperm rye } \\
\text { bread }\end{array}$ & 16 & $7.6 \mathrm{~g} / 50 \mathrm{~g} \mathrm{AC}$ & \multirow{2}{*}{$\begin{array}{l}\text { No difference in iAUC } C_{\text {cut }} \\
(240 \mathrm{~min}) \text { among breads }\end{array}$} & & $\begin{array}{l}\mathrm{iAUC} \text { cut }(\mathrm{mol} / / \times \mathrm{n} \\
938(\mathrm{SEM} 515)\end{array}$ & $\begin{array}{l}(240 \mathrm{~min}) \\
\text { WB) }\end{array}$ \\
\hline & White bread & & $3.7 \mathrm{~g} / 50 \mathrm{~g} \mathrm{AC}$ & & & 1297 (SEM & \\
\hline \multirow[t]{3}{*}{ Lappi et al..$^{(16)}$} & $\begin{array}{l}\text { Wholemeal wheat bread (100\% wheat flour } \\
\text { from peeled grains) }\end{array}$ & 11 IR subjects & $8.4 \mathrm{~g} / 50 \mathrm{~g} \mathrm{AC}$ & \multicolumn{2}{|l|}{$\begin{array}{l}\mathrm{iAUC} \text { cut }(\mathrm{mmol} / / \times \mathrm{min})(240 \mathrm{~min}) \\
204(\mathrm{sD} 86)\end{array}$} & \multicolumn{2}{|c|}{$\begin{array}{l}\mathrm{iAUC}_{\text {cut }}(\mathrm{mU} / \mathrm{m} / \mathrm{min})(240 \mathrm{~min}) \\
8064(\mathrm{sD} 4222)\end{array}$} \\
\hline & $\begin{array}{l}\text { Wholemeal wheat flour with xylanase } \\
\text { Wholemeal wheat flour with sourdough } \\
\text { (starter culture LA4) }\end{array}$ & & $\begin{array}{l}8.5 \mathrm{~g} / 50 \mathrm{~g} \mathrm{AC} \\
8.6 \mathrm{~g} / 50 \mathrm{~g} \mathrm{AC}\end{array}$ & \multicolumn{2}{|l|}{$\begin{array}{l}212(\mathrm{SD} 125) \\
173(\mathrm{SD} 105)\left(^{*} v . \mathrm{WB}\right)\end{array}$} & \multicolumn{2}{|c|}{$\begin{array}{l}8423(\text { sD } 4807) \\
7194(\text { SD } 3797)\end{array}$} \\
\hline & White wheat bread & & $3.3 \mathrm{~g} / 50 \mathrm{~g} \mathrm{AC}$ & \multicolumn{2}{|l|}{272 (sD 134) } & \multicolumn{2}{|c|}{8651 (sD 4097) } \\
\hline
\end{tabular}




\section{N British Journal of Nutrition}

\section{Table 1. Continued}

\begin{tabular}{|c|c|c|c|c|c|c|c|}
\hline \multirow[b]{2}{*}{ References } & \multirow[b]{2}{*}{ Bread formulation } & \multirow[b]{2}{*}{ Subjects† } & \multirow{2}{*}{$\begin{array}{l}\text { Total dietary } \\
\text { fibre content‡ }\end{array}$} & \multicolumn{2}{|c|}{ Glucose response§ } & \multicolumn{2}{|l|}{ Insulin response§ } \\
\hline & & & & iAUC & Gl & iAUC & ॥ \\
\hline $\begin{array}{l}\text { De Angelis } \\
\text { et al. }^{(13)}\end{array}$ & $\begin{array}{l}\text { Wheat sourdough and enriched with oat and } \\
\text { rye fibre }(71 \% \text { wheat flour, } 16 \% \\
\text { sourdough, } 9 \% \text { rye fibre, } 4 \% \text { oat fibre) }\end{array}$ & 20 & $8 \cdot 1$ & & $41 \cdot 0$ & & \\
\hline \multirow[t]{4}{*}{ Najjar et al. ${ }^{(14)}$} & Whole wheat bread $(71 \%$ whole wheat flour, & 10 & $6 \cdot 3 \mathrm{~g} / 50 \mathrm{~g} \mathrm{AC}$ & \multicolumn{2}{|l|}{$\begin{array}{l}\text { AUC }(\mathrm{mmol} / 1 \times \mathrm{min})(180 \mathrm{~min}) \\
167.7(\text { SEM } 32 \cdot 9)\end{array}$} & \multicolumn{2}{|l|}{$\begin{array}{l}\text { AUC }(\mathrm{nmol} / / \mathrm{l} \times \mathrm{min})(180 \mathrm{~min}) \\
39.2(\mathrm{SEM} 15.0)\end{array}$} \\
\hline & $\begin{array}{l}29 \% \text { whole-grain flour) } \\
\text { Sourdough bread ( } 100 \% \text { WWF, sourdough } \\
\text { starter) }\end{array}$ & overweight & $1.0 \mathrm{~g} / 50 \mathrm{~g} \mathrm{AC}$ & \multicolumn{2}{|l|}{$\begin{array}{l}96.3 \text { (SEM 31.3) }\left({ }^{*} v \text {. whole wheat }\right. \\
\text { bread) }\end{array}$} & \multicolumn{2}{|l|}{$37 \cdot 5$ (SEM 14.2) } \\
\hline & $\begin{array}{l}\text { Whole wheat and barley bread (72\% whole } \\
\text { wheat flour, } 28 \% \text { barley flour) }\end{array}$ & & $5.5 \mathrm{~g} / 50 \mathrm{~g} \mathrm{AC}$ & \multicolumn{2}{|l|}{$131 \cdot 7($ SEM $21 \cdot 0)$} & \multicolumn{2}{|l|}{33.9 (SEM 12.8) } \\
\hline & White bread & & $1.5 \mathrm{~g} / 50 \mathrm{~g} \mathrm{AC}$ & \multicolumn{2}{|l|}{124.0 (SEM 28.5) } & \multirow{2}{*}{\multicolumn{2}{|c|}{39.6 (SEM 15.0) }} \\
\hline Scazzina et al. ${ }^{(21)}$ & $\begin{array}{l}\text { Wholemeal bread with Saccharomyces } \\
\text { cerevisiae } \\
\text { Wholemeal bread with sourdough } \\
\text { WWF bread with S. cerevisiae } \\
\text { WWF bread with sourdough }\end{array}$ & 8 & $\begin{array}{l}10 \cdot 0 \\
9 \cdot 8 \\
2 \cdot 0 \\
2 \cdot 2\end{array}$ & \multicolumn{2}{|l|}{$\begin{array}{l}\text { Sourdough breads (both } \\
\text { wholemeal and white) showed } \\
\text { significantly lower iAUC } \\
\text { (120 min) than breads } \\
\text { leavened with baker's yeast }\end{array}$} & & \\
\hline \multirow[t]{2}{*}{$\begin{array}{l}\text { De Angelis } \\
\text { et al. }^{(15)}\end{array}$} & $\begin{array}{l}\text { Sourdough bread with oat fibre (1:1 wheat } \\
\text { flour and wholemeal flour) }\end{array}$ & 15 & & \multirow[t]{2}{*}{$\begin{array}{l}\text { IAUC (120 min) for sourdough } \\
\text { bread with oat fibre } \\
\text { significantly lower than that } \\
\text { of WB }\end{array}$} & $53 \cdot 7$ & & \\
\hline & White bread & & & & 72 & & \\
\hline \multirow[t]{4}{*}{$\begin{array}{l}\text { Juntunen } \\
\quad \text { et al. }\end{array}$} & $\begin{array}{l}100 \% \text { endosperm rye flour, sourdough } \\
\text { prepared from endosperm rye flour }\end{array}$ & 19 & $6.1 \mathrm{~g} / 50 \mathrm{~g} \mathrm{AC}$ & \multicolumn{2}{|l|}{$\begin{array}{l}\text { IAUC }(\mathrm{mmol} / 1 \times \mathrm{min})(180 \mathrm{~min}) \\
99.4(\text { SEM } 16.0)\end{array}$} & $\begin{array}{l}\text { iAUC }(\mathrm{pmol} / / \times \min )(180 \mathrm{~min}) \\
15831(\mathrm{sEM} 1276)\left({ }^{*} v . \mathrm{WB}\right)\end{array}$ & \\
\hline & $\begin{array}{l}100 \% \text { wholemeal rye flour, sourdough } \\
\text { prepared from wholemeal rye flour }\end{array}$ & & $15 \cdot 2 \mathrm{~g} / 50 \mathrm{~g} \mathrm{AC}$ & \multicolumn{2}{|l|}{77.8 (SEM 11.6$)$} & $16389\left(\right.$ SEM 1374) $\left({ }^{*} v\right.$. WB) & \\
\hline & $\begin{array}{l}60 \% \text { wholemeal rye flour }-40 \% \text { rye bran, } \\
\text { sourdough prepared from wholemeal } \\
\text { rve flour }\end{array}$ & & $29.0 \mathrm{~g} / 50 \mathrm{~g} \mathrm{AC}$ & \multicolumn{2}{|l|}{$83 \cdot 3$ (SEM 23.3) } & \multicolumn{2}{|l|}{18270 (SEM 1755) } \\
\hline & White bread & & $2.7 \mathrm{~g} / 50 \mathrm{~g} \mathrm{AC}$ & \multicolumn{2}{|l|}{99.6 (SEM 15.1) } & \multicolumn{2}{|l|}{22151 (SEM 2288) } \\
\hline
\end{tabular}

iAUC, incremental AUC; GI, glycaemic index; II, insulinaemic index; T2D, type 2 diabetes; AC, available carbohydrates; WB, white bread; IR, insulin resistant; WWF, white wheat flour. * Statistical significance, $P<0.05$

† Subjects are healthy individuals, if not, further details are given.

$\neq$ Unless otherwise stated, total dietary fibre content corresponds to $\mathrm{g} / 100 \mathrm{~g}$ of bread.

§ More information about how AUC, iAUC, iAUCcut and net iAUC are calculated can be found in the article by Brouns et al.(44). Gl and II values are expressed with G as the reference, being assigned the value of 100 .

$\|$ Time point -15 was used as the baseline, and values below the baseline were considered to be negative peaks. Data were log transformed before statistical analysis. 


\section{N British Journal of Nutrition}

Table 2. Selected sourdough details of sourdough breads

(Mean values and standard deviations)

\begin{tabular}{|c|c|c|c|c|c|c|c|c|c|c|c|}
\hline \multirow[b]{2}{*}{ References } & \multirow[b]{2}{*}{ Type of Bread } & \multicolumn{2}{|c|}{$\mathrm{pH}$} & \multicolumn{2}{|c|}{ Titratable acidity } & \multirow[b]{2}{*}{ Starter culture } & \multicolumn{2}{|c|}{ Lactic acid } & \multicolumn{2}{|c|}{ Acetic acid } & \multirow{2}{*}{$\begin{array}{l}\text { Sourdough } \\
\text { addition level }\end{array}$} \\
\hline & & Sourdough & Bread & Sourdough & Bread & & Sourdough & Bread & Sourdough & Bread & \\
\hline \multirow[t]{4}{*}{ Tucker et al. (22) } & $\begin{array}{l}\text { Three-grain sprouted } \\
\text { sourdough bread }\end{array}$ & & $4 \cdot 20$ & & & & & & & & \\
\hline & $\begin{array}{l}\text { Whole-grain wheat } \\
\text { (predominately), rye } \\
\text { sourdough bread }\end{array}$ & & 4.70 & & & & & & & & \\
\hline & $\begin{array}{l}\text { White flour bread with lactic } \\
\text { acid, ascorbic acid, } \\
\text { sodium acetate }\end{array}$ & & $4 \cdot 10$ & & & & & & & & \\
\hline & White bread & & 4.70 & & & & & & & & \\
\hline Novotni et al. ${ }^{(32)}$ & $\begin{array}{l}\text { Gluten free breads with: } \\
0 \% \text { sourdough } \\
7.5 \% \text { sourdough } \\
15 \% \text { sourdough } \\
22.5 \% \text { sourdough } \\
30 \% \text { sourdough }\end{array}$ & 4.01 (sD 0.05) & $\begin{array}{l}5.96(\text { sD } 0.01) \\
5.89(\text { sD } 0.08) \\
5.73(\text { sD } 0.04) \\
5.58(\text { SD } 0.04) \\
5.40(\text { sD } 0.04)\end{array}$ & $4.0 \dagger$ & & PL3 (Lactobacillus fermentum) & $\begin{array}{c}(\mathrm{g} / 100 \mathrm{~g}) \\
0.171(\mathrm{sD} 0.010)\end{array}$ & $\begin{array}{c}(\mathrm{g} / 100 \mathrm{~g}) \\
<0.01 \\
0.022(\mathrm{sD} 0.003) \\
0.045(\mathrm{sD} 0.004) \\
0.064(\mathrm{sD} 0.004) \\
0.087(\mathrm{sD} 0.007)\end{array}$ & $\begin{array}{c}(\mathrm{g} / 100 \mathrm{~g}) \\
0.024(\mathrm{sD} 0.004)\end{array}$ & $\begin{array}{c}(\mathrm{g} / 100 \mathrm{~g}) \\
0.013(\mathrm{sD} 0.001) \\
0.016(\mathrm{sD} 0.004) \\
0.018(\mathrm{sD} 0.002) \\
0.019 \text { (sD } 0.001) \\
0.019 \text { (sD } 0.004)\end{array}$ & $\begin{array}{c}0 \% \\
7.5 \% \\
15 \% \\
22.5 \% \\
30 \%\end{array}$ \\
\hline \multirow[t]{2}{*}{ Novotni et al. ${ }^{(33)}$} & $\begin{array}{l}\text { Wholemeal wheat PBF } \\
\text { breads prepared with }\end{array}$ & 4.0 & & & & $\begin{array}{l}\text { Lactobacillus plantarum } \\
\text { (pure culture) }\end{array}$ & $\begin{array}{c}(\mathrm{g} / 100 \mathrm{~g}) \\
0.96(\mathrm{sD} 0.06)\end{array}$ & & $\begin{array}{c}(\mathrm{g} / 100 \mathrm{~g}) \\
0.01(\mathrm{sD} 0.01)\end{array}$ & & $10 \%$ \\
\hline & different starter cultures & & & & & $\begin{array}{l}\text { L. fermentum (PL1) } \\
\text { L. fermentum with phytase (PL3) } \\
\text { Lactobacillus brevis combined } \\
\text { with S. chevalieri (LV4) }\end{array}$ & $\begin{array}{l}0.88(\text { sD } 0.05) \\
0.93(\text { SD } 0.09) \\
0.67(\text { SD } 0.06)\end{array}$ & & $\begin{array}{l}0.15(\text { sD } 0.03) \\
0.14(\text { sD } 0.05) \\
0.19(\text { sD } 0.04)\end{array}$ & & $\begin{array}{l}10 \% \\
10 \% \\
10 \%\end{array}$ \\
\hline \multirow[t]{3}{*}{ Lappi et al..$^{(16)}$} & $\begin{array}{l}\text { Wholemeal wheat bread } \\
\text { Wholemeal wheat flour with } \\
\text { xylanase }\end{array}$ & $3 \cdot 9-4 \cdot 1$ & $\begin{array}{l}6 \cdot 0 \\
6 \cdot 0\end{array}$ & $16-17$ & $\begin{array}{c}3.8 \ddagger \\
3.8\end{array}$ & LA4 & & & & & \\
\hline & $\begin{array}{l}\text { Wholemeal wheat flour with } \\
\text { sourdough }\end{array}$ & & 4.6 & & 11.5 & & & & & & \\
\hline & White wheat bread & & 5.9 & & $2 \cdot 3$ & & & & & & \\
\hline De Angelis et al..$^{(13)}$ & $\begin{array}{l}\text { Wheat sourdough and } \\
\text { enriched with oat and } \\
\text { rye fibre }\end{array}$ & 3.8 (sD 0.13$)$ & & & & $\begin{array}{l}\text { Lactobacillus sanfranciscensis } \\
\text { DPPMA12, L. plantarum } \\
\text { DPPMA55 and Lactobacillus } \\
\text { sp. DPPMA56 }\end{array}$ & $\begin{array}{c}(\mathrm{mM}) \\
130.0(\mathrm{sD} 4 \cdot 20)\end{array}$ & & $\begin{array}{c}(\mathrm{mm}) \\
42 \cdot 0(\mathrm{sD} 2 \cdot 10)\end{array}$ & & $10 \%$ \\
\hline \multirow[t]{2}{*}{ Naijar et al. ${ }^{(14)}$} & $\begin{array}{l}\text { Sourdough bread ( } 100 \% \\
\text { white wheat flour, } \\
\text { sourdough starter) }\end{array}$ & & 5.07 & & & & & & & & $27 \%$ \\
\hline & White bread & & 5.77 & & & & & & & & \\
\hline \multirow[t]{4}{*}{ Scazzina et al. ${ }^{(21)}$} & $\begin{array}{l}\text { Wholemeal bread with } \\
\text { S. cerevisiae }\end{array}$ & & & & $85 \S$ & & & & & & \\
\hline & $\begin{array}{l}\text { Wholemeal bread with } \\
\text { sourdough }\end{array}$ & & & & 122 & & & & & & \\
\hline & $\begin{array}{l}\text { White wheat bread with } \\
\text { S. cerevisiae }\end{array}$ & & & & 53 & & & & & & \\
\hline & $\begin{array}{l}\text { White wheat bread with } \\
\text { sourdough }\end{array}$ & & & & 77 & & & & & & \\
\hline De Angelis et al..$^{(15)}$ & $\begin{array}{l}\text { Sourdough bread with } \\
\text { oat fibre }\end{array}$ & & & & & L. plantarum $\mathrm{P} 1$ and $L$. brevis $\mathrm{P} 2$ & & & & & \\
\hline \multirow[t]{2}{*}{ Juntunen et al. ${ }^{(19)}$} & Endosperm rye bread & & & & & $\begin{array}{l}\text { L62 (L. brevis), L73 } \\
\text { (L. plantarum) }\end{array}$ & & & & & $35 \%$ \\
\hline & $\begin{array}{l}\text { Traditional rye bread } \\
\text { High-fibre rye bread }\end{array}$ & & & & & & & & & & $\begin{array}{l}35 \% \\
34 \%\end{array}$ \\
\hline
\end{tabular}

PBF, partially baked frozen.

*Sourdough addition levels correspond to $\mathrm{g}$ of sourdough per $100 \mathrm{~g}$ of bread dough.

$\dagger \mathrm{ml} \mathrm{NaOH} 0.1 \mathrm{~m}$ used for titration until pH 8.5.

$\ddagger \mathrm{ml} \mathrm{NaOH} 0.1 \mathrm{M}$ used to titrate $10 \mathrm{~g}$ of bread.

$\S$ Milliequivalents of $\mathrm{H}^{+} / \mathrm{kg}$. 
types, eleven-grain bread (whole grain with sourdough culture), sprouted-grain bread (whole grain with sourdough culture), sourdough white bread, twelve-grain bread (whole grain) and white wheat bread. The main finding was that sprouted grain bread lowered glucose responses, which could be attributed to the increased dietary fibre content and increased availability of $\mathrm{mg}$, vitamin $\mathrm{E}$, phenolic compounds and phytoestrogens possibly able to act synergistically to lower glycaemia.

\section{Suggested mechanisms of sourdough action}

In an attempt to explore the mechanisms underlying the lower metabolic responses to sourdough bread consumption, gastric emptying rate has been examined by several studies ${ }^{(24-25)}$. Darwiche et al. ${ }^{(25)}$ using ultrasonography showed a decrease in the gastric emptying rate of barley bread containing sodium propionate, which caused lower glucose and insulin levels compared with plain barley bread. Similar results were obtained by Liljeberg \& Bjorck $^{(26)}$ when bread was enriched with sodium propionate but not with lactic acid. However, Najjar et al. ${ }^{(14)}$, who examined gastric emptying rate by adding paracetamol in the flour of the breads, did not detect any differences between white bread and sourdough white bread. In agreement to the previous study, no difference in gastric emptying rate was reported in the study by Bondia-Pons et al. ${ }^{(20)}$ among endosperm sourdough rye bread and white bread. The involved mechanisms remain to be elucidated.

Sourdough fermentation is hypothesised to increase the resistant starch (RS) content of breads. Breads made of white wheat flour and wholemeal flour and fermented with selected sourdough L. plantarum and L. brevis strains contain higher concentrations of RS (approximately 5\%) than breads started with baker's yeast alone (RS: $1.4-1.7 \%)^{(15)}$. In accordance, breads prepared with white or wholemeal wheat flour and leavened with sourdough were found to have higher amounts of RS compared with same breads leavened with $S$. cerevisiae. Liljeberg et $a l .{ }^{(26)}$ observed an increase of RS content in breads containing increasing concentrations of lactic acid, leading to the hypothesis that the presence of organic acids in bread may increase starch retrogradation and thus RS content.

It has been also reported that sourdough fermented breads may result in lower rate of starch hydrolysis ${ }^{(27)}$. De Angelis et $a l .{ }^{(15)}$ reported that the rate of starch hydrolysis of breads containing lactic and acetic acids was lower compared with non-acidified breads in vitro. Furthermore, comparing microbial acidification by selected sourdough lactobacilli with chemical acidification in terms of rate of starch hydrolysis, breads fermented with sourdough lactobacilli cause a significantly greater effect. Opposite results are demonstrated by Scazzina et $a l^{(21)}$, who supported that the leavening technique does not influence starch digestibility or availability to hydrolytic enzymes. In this study no difference in rate of starch digestion in vitro was found between white or wholemeal breads fermented either by sourdough microflora or by yeast.

Another approach suggests that proteolysis, which takes place during sourdough fermentation ${ }^{(28)}$, may contribute to lowered postprandial glucose responses. Lappi et al. ${ }^{(16)}$ analysed the state of protein in an attempt to explain the mechanisms underlying the beneficial postprandial responses to sourdough fermented bread. Although in vitro analysis revealed no difference in the hydrolysis rate of protein among the sourdough and other wholemeal wheat breads, the content of soluble proteins was higher and their molecular weights was lower in sourdough fermented breads, suggesting that more solubilized proteins are ingested in the case of sourdough fermented wholemeal wheat bread. Although a higher insulin response could be expected after the sourdough bread consumption due to stimulation of insulin secretion from the protein degradation by sourdough fermentation, a trend for lower insulin response was observed.

It is possible, that the sourdough fermentation increases the interaction between starch and gluten proteins of cereals, resulting in the creation of a barrier which limits starch bioavailability and enzyme accessibility. The effect is positive if the acid is present during the gelatinisation of starch but not after the thermal treatment ${ }^{(27)}$. Other effects of sourdough leavening appear to be the synthesis of free phenolic compounds which are thought to improve glucose tolerance ${ }^{(29-31)}$.

\section{Sourdough fermentation in combination with partial baking-freezing technology}

Sourdough fermentation technology was recently combined with partially baking freezing technology for the production of low GI breads and gluten-free breads ${ }^{(32-33)}$. Partial-baking freezing technology involves initial baking equivalent to $75 \%$ of total baking time, followed by freezing, storage and final baking, and makes freshly baked bread available at any time. Novotni et al. ${ }^{(33)}$ used four different lactic acid bacteria starters to ferment sourdough for the production of partially baked frozen wholemeal wheat bread. Five partially baked frozen breads were prepared and tested for postprandial glucose response by ten healthy volunteers in a cross-over design study (glucose solution was used as reference). Breads included a control wholemeal bread without sourdough, a bread prepared with $L$. plantarum sourdough (10\%), another one with PL1 (Lactobacillus fermentum) sourdough (10\%), a bread with PL3 (L. fermentum with phytase) sourdough (10\%) and finally a bread prepared with LV4 ( $L$. brevis combined with $S$. cerevisiae var. chevalieri) sourdough (10\%). Their GI values were calculated 70, 60, 56, 56 and 50, respectively. The bread prepared with $L$. plantarum sourdough did not result in a significantly lower GI compared with control partially baked bread without sourdough. These results suggest that partially baked frozen sourdough bread with a reduced GI can be prepared using L. brevis and L. fermentum starters. This combined technology was also used for the development of low GI gluten-free breads ${ }^{(32)}$. Commercial starter PL3 (L. fermentum combined with phytase) was used in four levels, $7 \cdot 5,15,22.5$ and $30 \mathrm{~g}$ of sourdough/100 g of bread and a control also gluten-free bread without sourdough was prepared. In all, eleven healthy subjects participated in this cross-over design trial and consumed in six separate occasions the five test breads and a glucose solution as reference food. The results showed that sourdough decreased bread's GI by 9, 16, 14 or 7 units, respectively. Interestingly, the highest amount of sourdough gave the smallest reduction in bread's GI. The researchers attributed this 
result to the observed decrease of bread viscosity that could have caused an increased postprandial glucose response, and recommended that sourdough should be added in moderate amounts in order to deliver the desired outcome.

\section{Other processing conditions}

Apart from the leavening technique used for bread making, alteration of other bread processing conditions is another strategy that could be used for the improvement of white bread's GI. Processing can modify the microstructure, composition and availability of different compounds, including starch. The reviewed studies are presented on Table 3.

It has been recently shown by Lau et al. ${ }^{(5)}$, that even when the same ingredients are used, the application of varied processing procedures including mixing time, mixing intensity, proofing period and method of cooking can significantly influence postprandial glucose response to white bread. In particular, four types of white bread were prepared, a Western baked bread (high protein wheat flour, proofing time $70 \mathrm{~min}$, baked at $210^{\circ} \mathrm{C}$ for $11 \mathrm{~min}$ ), an oriental steamed bread, popular staple in Asia (medium protein wheat flour, proofing time $40 \mathrm{~min}$, baked at $100^{\circ} \mathrm{C}$ for $10 \mathrm{~min}$ ), a modified baked bread and a modified steamed bread (both with the recipe of Western bread and oriental processing conditions). The ingestion of these four breads by healthy subjects allowed the calculation of their GI values, which were seventy-five for modified baked bread, seventy-one for Western baked bread, sixty-eight for oriental steamed bread and sixty-five for modified steamed bread. Differences in processing procedures caused differences in physical structure, which in turn resulted in starch digestibility differences. It was assumed that steaming resulted in a more compact structure which could inhibit the accessibility of amylase to starch granules leading to a slower rate of glucose release and therefore a lower GI.

Pumpernickel breads are commonly consumed in Germany and Scandinavia and constitute a healthy alternative to wheat bread for T2D patients. They are usually baked for $20 \mathrm{~h}$ at $120^{\circ} \mathrm{C}$, and as a result they contain high amounts of RS and a mechanically firmer and denser structure. Low processing temperature can block the hydration and swelling of starch granules hindering starch gelatinisation and facilitating the formation of $\mathrm{RS}^{(34)}$. Indeed, in the study by Breen et $a l^{(35)}$ a pumpernickel rye bread, was shown to promote lower postprandial glucose and insulin responses in T2D patients, compared both with white bread and whole wheat grain bread.

The manipulation of dough proofing, resulting in different loaf volume, was examined in the study by Burton \& Lightowler $^{(36)}$. In all, ten healthy subjects participated in a randomised trial and consumed four slices of four breads differing in volume but not in macronutrient composition (glucose was used as reference). The volumes of bread loaves were 1100, 1700, 2400 and $3000 \mathrm{ml}$. GI values were shown to be significantly reduced by lowering loaf volume, and were found 38, 72, 86 and 100, respectively. This study suggests that reducing bread volume is accompanied by a drop in bread's GI. The postprandial glucose response to a compact and dense bread made from candeal-flour (poor in gluten), prepared with short fermentation time and commonly consumed in Spain was evaluated in the study by Gonzalez-Anton et al. ${ }^{(37)}$. The GI was calculated 86 (with glucose as reference) showing no difference compared with white bread $(\mathrm{GI}=76)$, but II was found lower compared with reference.

The effect of different rising methods and leavening agents on bread's GI was studied in the study by Fredensborg et $a l .{ }^{(38)}$. Long fermentation ( $50 \mathrm{~min}$ rise and proofing time) breads were compared with short fermented breads (30 min proofing time), but no difference in GI value was detected. In addition, the use of different leavening agents were compared, yeast, desem (fermented dough, in Dutch) and sourdough, but again they did not significantly affect the GI of bread. These results suggest that increasing the fermentation time by approximately $20 \mathrm{~min}$ does not sufficiently influence the GI of bread, and possibly other factors are required for the design of low GI breads. Rizkalla et $a l .{ }^{(39)}$ demonstrated a comparison between postprandial plasma glucose and insulin responses after the ingestion of a variety of French breads differing in baking process. Test breads were classic baguette, traditional baguette, loaf of wholemeal bread, loaf of bread fermented with yeast or with leaven and a glucose challenge as reference, and consumed in amounts that contained $50 \mathrm{~g}$ of available carbohydrates. Results showed that GI values ranged from 57, for the traditional baguette, to 85 for the wholemeal bread, whereas no significant difference was detected among them. However, the II of the traditional baguette and of the bread fermented with leaven were lower than the other breads. These results were attributed to the long fermentation time and production of organic acids for the bread fermented with leaven, and to the artisanal preparing method that prevented the dough to rise at its maximal capacity, for the traditional baguette.

The application of frozen storage on baking technology has been shown to favourably alter the GI of white bread. A randomised controlled cross-over design trial by Borczak et al. ${ }^{(40)}$ studied the effect of both fibre addition and freezing treatment in white-flour wheat rolls on postprandial blood glucose in human volunteers. Healthy subjects consumed four types of wheat rolls which included (1) fully baked, non-frozen, (2) fully baked, non-frozen with dietary fibre (10\%), (3) partially baked and frozen and (4) partially baked and frozen with dietary fibre (10\%), in amounts that yielded $50 \mathrm{~g}$ of available carbohydrates. The results showed that both the addition of dietary fibre and freezing applied to wheat rolls significantly reduced the GI by $34 \%$ (GI of partially baked frozen with dietary fibre was 53 with glucose as reference food) compared with fully baked without dietary fibre wheat roll $(\mathrm{GI}=87)$. Another study by the same research group, examined the effect of sourdough addition to partially baked and frozen wheat rolls on bread's $\mathrm{GI}^{(41)}$. For this purpose, four test wheat rolls were tested by healthy subjects: fully baked non-frozen, fully baked non-frozen with 3\% dehydrated sourdough, partially baked frozen, partially baked frozen with $3 \%$ dehydrated sourdough. The GI values of the products were calculated 87, 63, 67 and 43, respectively. Both factors, freezing and sourdough, significantly reduced the GI of wheat rolls. Heating-cooling cycles that are present during partially baking freezing technology result in the formation of 
Table 3. Human studies examining the effect of other processing conditions on postprandial glucose $(G)$ and insulin response (Mean values and standard errors; mean values and standard deviations; mean values and $95 \%$ confidence intervals)

\begin{tabular}{|c|c|c|c|c|c|c|c|}
\hline \multirow[b]{2}{*}{ References } & \multirow[b]{2}{*}{ Bread formulation } & \multirow[b]{2}{*}{ Subjects† } & \multirow[b]{2}{*}{$\begin{array}{l}\text { Total dietary fibre } \\
\text { content\$ }\end{array}$} & \multicolumn{2}{|c|}{ Glucose response§ } & \multicolumn{2}{|c|}{ Insulin response§ } \\
\hline & & & & iAUC & Gl & IAUC & II \\
\hline \multirow[t]{6}{*}{$\begin{array}{l}\text { Gonzalez-Anton } \\
\text { et al. }{ }^{(37)}\end{array}$} & & & & $\begin{array}{l}\operatorname{AUC}(\mathrm{mg} / \mathrm{dl} \times \mathrm{min}) \\
(120 \mathrm{~min})\end{array}$ & & $\begin{array}{l}\mathrm{AUC}(\mathrm{mU} / \mathrm{ml} \times \mathrm{min}) \\
(120 \mathrm{~min})\end{array}$ & \\
\hline & Ordinary white bread & 22 & 4.1 & 910 (SEM 125) & 76 & 2020 (SEM 247) & $68\left({ }^{*} v . \mathrm{G}\right)$ \\
\hline & Precooked frozen white bread & & 4.3 & 854 (SEM 135) & 78 & 1810 (SEM 254) & $68\left({ }^{*} v \cdot \mathrm{G}\right)$ \\
\hline & Candeal-flour white bread & & 3.3 & 1133 (SEM 127) & & 1955 (SEM 248) & $69\left({ }^{*} v . \mathrm{G}\right)$ \\
\hline & Alfacar white bread & & 3.5 & 886 (SEM 125) & $68\left({ }^{*} v . \mathrm{G}\right)$ & 1939 (SEM 248) & $70\left({ }^{*} v . \mathrm{G}\right)$ \\
\hline & Wholemeal bread & & 7.9 & 828 (SEM 125) & $61\left({ }^{*} v . \mathrm{G}\right)$ & 2190 (SEM 248) & $73\left({ }^{*} v . G\right)$ \\
\hline \multirow[t]{4}{*}{ Lau et al. ${ }^{(5)}$} & & & & $\begin{array}{l}\mathrm{iAUC}(\mathrm{mmol} / \mathrm{l} \times \mathrm{min}) \\
(120 \mathrm{~min})\end{array}$ & & & \\
\hline & $\begin{array}{l}\text { WBB } \\
\text { OSB }\end{array}$ & 13 & Not provided & $\begin{array}{l}196(\text { SEM 17) } \\
184(\text { SEM 13) }\end{array}$ & $\begin{array}{l}71(\text { SEM 5) } \\
68(\text { SEM 5) }\end{array}$ & & \\
\hline & $\begin{array}{l}\text { Modified baked bread, with OSB recipe and WBB } \\
\text { processing conditions }\end{array}$ & & & 211 (SEM 18) & 75 (SEM 4) & & \\
\hline & $\begin{array}{l}\text { Modified steamed bread, with WBB recipe and OSB } \\
\text { processing conditions }\end{array}$ & & & 179 (SEM 13) & 65 (SEM 4) & & \\
\hline \multirow[t]{4}{*}{ Breen et al. ${ }^{(35)}$} & & & & $\begin{array}{l}\mathrm{iAUC}(\mathrm{mg} / \mathrm{dl} \times \mathrm{min}) \\
(270 \mathrm{~min})\end{array}$ & & $\begin{array}{l}\mathrm{iAUC}(\mathrm{uU} / \mathrm{ml} \times \mathrm{min}) \\
(270 \mathrm{~min})\end{array}$ & \\
\hline & Pumpernickel rye bread & $\begin{array}{l}11 \mathrm{~T} 2 \mathrm{D} \\
\text { patients }\end{array}$ & $19 \cdot 2 \mathrm{~g} / 50 \mathrm{~g} \mathrm{AC}$ & $\begin{array}{l}6570 \text { (sD 2426.4) ( }{ }^{*} v . \\
\text { whole grain) }\end{array}$ & & $\begin{array}{l}3522 \cdot 8\left({ }^{*} v .\right. \\
\text { WB }+ \text { whole grain) }\end{array}$ & \\
\hline & $\begin{array}{l}\text { Whole wheat bread (whole wheat flour and buttermilk) } \\
\text { Whole-grain bread (whole wheat flour and } \\
\text { cracked wheat) }\end{array}$ & & $\begin{array}{l}7.4 \mathrm{~g} / 50 \mathrm{~g} \mathrm{AC} \\
7.5 \mathrm{~g} / 50 \mathrm{~g} \mathrm{AC}\end{array}$ & $\begin{array}{l}7826 \cdot 4(\text { SD 1960.2) } \\
8476 \cdot 2 \text { (SD 1718.8) }\end{array}$ & & $\begin{array}{l}5603.9 \\
10991.6\end{array}$ & \\
\hline & White bread & & $3.4 \mathrm{~g} / 50 \mathrm{~g} \mathrm{AC}$ & $8022 \cdot 6$ (sD 2073.6) & & 10 533.8 (sD 4126.4) & \\
\hline \multirow[t]{5}{*}{ Borczak et al. ${ }^{(40)}$} & & & & $\begin{array}{l}\underset{\mathrm{iAUC}}{(120 \mathrm{mmol} / / \mathrm{l} \times \mathrm{min})} \\
\quad\end{array}$ & & & \\
\hline & White wheat bread & 15 & & & & & \\
\hline & $\begin{array}{l}\text { FBNF } \\
\text { FBNF }+10 \% \text { dietary fibre }\end{array}$ & & $\begin{array}{l}2 \cdot 9 \\
7 \cdot 8\end{array}$ & 161 (SEM 19) & 87 (SEM 11) & & \\
\hline & $\begin{array}{l}\text { PBNF }+10 \% \text { dietary ribre } \\
\text { PBF }\end{array}$ & & $\begin{array}{l}7.8 \\
3.2\end{array}$ & $\begin{array}{l}130 \text { (SEM 16) }\left({ }^{*} V . G\right) \\
123\left(\text { SEM 15) }\left({ }^{*} V . G\right)\right.\end{array}$ & $\begin{array}{l}72(\text { SEM 6) } \\
67 \text { (SEM 6) }\end{array}$ & & \\
\hline & PBF $+10 \%$ dietary fibre & & $7 \cdot 9$ & 106 (SEM 14) (*v. G) & 53 (SEM 7) (* v. FBNF) & & \\
\hline \multirow[t]{4}{*}{ Borczak et al. ${ }^{(41)}$} & White wheat bread & 15 & & $\begin{array}{l}\mathrm{iAUC}(\mathrm{mmol} / \mathrm{l} \times \mathrm{min}) \\
(120 \mathrm{~min})\end{array}$ & & & \\
\hline & FBNF & & 2.9 & 161 (SEM 16) & 87 (SEM 11) & & \\
\hline & $\begin{array}{l}\text { FBNF }+3 \% \text { dehydrated sourdough } \\
\mathrm{PBF}\end{array}$ & & $\begin{array}{l}3 \cdot 2 \\
2.4\end{array}$ & $111\left(\right.$ SEM 12) $\left({ }^{*} V \cdot G\right)$ & 63 (SEM 7) $\left(^{*} v\right.$. FBNF) & & \\
\hline & PBF + $3 \%$ dehydrated sourdough & & 3.2 & 86 (SEM 12) ( $\left.{ }^{*} V . \mathrm{G}\right)$ & $\begin{array}{l}43 \text { (SEM } 4)\left(^{*} v \text {. FBNF) }\right. \\
\text { PBF) }\end{array}$ & & \\
\hline $\begin{array}{l}\text { Fredensborg } \\
\text { et al. }{ }^{(38)}\end{array}$ & $\begin{array}{l}\text { Short rye bread } \| \\
\text { Short oat bread } \\
\text { Short whole wheat bread } \\
\text { Long rye bread|| } \\
\text { Long oat bread } \\
\text { Long whole wheat bread } \\
\text { Yeast whole wheat bread } \\
\text { Yeast + desem whole wheat bread } \\
\text { Sourdough whole wheat bread } \\
\text { Sourdough + oats whole wheat bread } \\
\text { Swiss Rye bread (commercial) }\end{array}$ & $\begin{array}{l}3 \text { groups of } \\
10\end{array}$ & $\begin{array}{l}4.8 \\
5 \cdot 2 \\
4.7 \\
6.9 \\
6.6 \\
4.6 \\
7.5 \\
7.3 \\
7.8 \\
4.7 \\
5.5\end{array}$ & & $\begin{array}{l}82(95 \% \mathrm{Cl} 57,107) \\
77(95 \% \mathrm{Cl} 63,92) \\
78(95 \% \mathrm{Cl} 66,90) \\
76(95 \% \mathrm{Cl} 63,30) \\
68(95 \% \mathrm{Cl} 52,84) \\
80(95 \% \mathrm{Cl} 48,112) \\
88(95 \% \mathrm{Cl} 70,107) \\
92(95 \% \mathrm{Cl} 69,116) \\
82(95 \% \mathrm{Cl} 53,111) \\
71(95 \% \mathrm{Cl} 48,94) \\
60(95 \% \mathrm{Cl} 54,66)\end{array}$ & & \\
\hline
\end{tabular}




\section{Bs British Journal of Nutrition}

Table 3. Continued

\begin{tabular}{|c|c|c|c|c|c|c|c|}
\hline \multirow[b]{2}{*}{ References } & \multirow[b]{2}{*}{ Bread formulation } & \multirow[b]{2}{*}{ Subjects $\dagger$} & \multirow[b]{2}{*}{$\begin{array}{l}\text { Total dietary fibre } \\
\text { content } \neq\end{array}$} & \multicolumn{2}{|c|}{ Glucose response§ } & \multicolumn{2}{|c|}{ Insulin response§ } \\
\hline & & & & iAUC & Gl & IAUC & II \\
\hline \multirow[t]{7}{*}{$\begin{array}{l}\text { Burton \& } \\
\text { Lightowler }^{(42)}\end{array}$} & & & & $\begin{array}{l}\mathrm{iAUC}(\mathrm{mmol} / \mathrm{l} \times \mathrm{min}) \\
\quad(120 \mathrm{~min})\end{array}$ & & & \\
\hline & $\begin{array}{l}\text { Homemade white bread: } \\
\text { Fresh }\end{array}$ & 10 & 1.9 & 259 (SD 103) & & & \\
\hline & Frozen and defrosted & & & $\begin{array}{l}179(\mathrm{sD} 74) \\
(* \text { v. G+ fresh) }\end{array}$ & & & \\
\hline & Fresh, toasted & & & $\begin{array}{l}193(\mathrm{sD} 79) \\
\quad\left({ }^{*} v \cdot \mathrm{G}+\text { fresh }\right)\end{array}$ & & & \\
\hline & Toasted following freezing and defrosting & & & $\begin{array}{l}157(\mathrm{SD} 85) \\
\quad(* \text { v. G+fresh) }\end{array}$ & & & \\
\hline & $\begin{array}{l}\text { Commercial white bread: } \\
\text { Fresh } \\
\text { Frozen and defrosted } \\
\text { Fresh, toasted }\end{array}$ & & 3.1 & $\begin{array}{l}253(\mathrm{SD} 106) \\
217(\mathrm{SD} 99)\left({ }^{*} v . \mathrm{G}\right) \\
183(\mathrm{SD} 96) \\
\quad\left({ }^{*} \text { v. G }+ \text { fresh }\right)\end{array}$ & & & \\
\hline & Toasted following freezing and defrosting & & & $\begin{array}{l}187(\mathrm{SD} 95) \\
\quad\left({ }^{*} v \cdot \mathrm{G}+\text { fresh }\right)\end{array}$ & & & \\
\hline \multirow[t]{3}{*}{ Rizkalla et al. ${ }^{(39)}$} & $\begin{array}{l}\text { Classic French baguette } \\
\text { Traditional French baguette }\end{array}$ & 9 & $\begin{array}{l}2 \cdot 9 \\
2 \cdot 3\end{array}$ & & $\begin{array}{l}78 \text { (SEM 17) } \\
57 \text { (SEM 9) }\end{array}$ & & $\begin{array}{l}90 \text { (SEM 15) } \\
50 \text { (SEM } 7)\left({ }^{\star} v \text {. classic }\right. \\
\text { baguette) }\end{array}$ \\
\hline & Wholemeal bread & & $5 \cdot 6$ & & 85 (SEM 27) & & 78 (SEM 21) \\
\hline & $\begin{array}{l}\text { Bread termented with yeast } \\
\text { Bread fermented with leaven }\end{array}$ & & $2 \cdot 1$ & & 80 (SEM 18) & & $\begin{array}{c}59 \text { (SEM } 7)\left({ }^{*} v \text {. classic }\right. \\
\text { baguette) }\end{array}$ \\
\hline \multirow[t]{4}{*}{$\begin{array}{l}\text { Burton \& } \\
\text { Lightowler }^{(36)}\end{array}$} & $\begin{array}{l}4 \text { bread slices of white bread }(50 \mathrm{~g} \mathrm{AC}) \text { of different } \\
\text { volume: }\end{array}$ & 10 & Not provided & $\begin{array}{l}\mathrm{iAUC}(\mathrm{mmol} / \mathrm{l} \times \mathrm{min}) \\
\quad(120 \mathrm{~min})\end{array}$ & & & \\
\hline & $\begin{array}{l}3000(\text { SD 150) } \mathrm{ml} \\
2400(\mathrm{SD} \mathrm{150)} \mathrm{ml}\end{array}$ & & & $\begin{array}{l}273 \text { (SEM 30) } \\
230 \text { (SEM 30) }\end{array}$ & $\begin{array}{l}100(\text { SEM } 7) \\
86\left(\text { SEM 9) }\left({ }^{*} v \text {. bread of }\right.\right. \\
300 \mathrm{ml})\end{array}$ & & \\
\hline & $1700(\mathrm{sD} 150) \mathrm{ml}$ & & & 204 & $\begin{array}{l}72\left({ }^{*} \text { v. bread of }\right. \\
3000 \mathrm{ml}\end{array}$ & & \\
\hline & $1100(\mathrm{sD} 100) \mathrm{ml}$ & & & 106 (SEM 15) & $\begin{array}{l}38\left(\text { SEM 4) }\left({ }^{*} V \text {. bread of }\right.\right. \\
3000 \mathrm{ml})\end{array}$ & & \\
\hline \multirow[t]{2}{*}{$\begin{array}{l}\text { Johnson } \\
\text { et al. }\end{array}$} & $76 \%$ WWF - $24 \%$ chickpea flour & 11 & $5.0 \mathrm{~g} / 50 \mathrm{~g} \mathrm{AC}$ & & 78 (SEM 13) (WB as ref) & & 147 (SEM 13) \\
\hline & $76 \%$ WWF - $24 \%$ extruded chickpea flour & & $6.0 \mathrm{~g} / 50 \mathrm{~g} \mathrm{AC}$ & & 96 (SEM 21) (WB as ref) & & $\begin{array}{l}120 \text { (SEM 14) } \\
\text { (WB as ref) }\end{array}$ \\
\hline
\end{tabular}

iAUC, incremental AUC; Gl, glycaemic index; II, insulinaemic index; WBB, Western baked bread; OSB, oriental steamed bread; T2D, type 2 diabetes; AC available carbohydrates; WB, white bread; FBNF, fresh baked non-frozen; $\mathrm{PBF}$, partially baked, frozen; WWF, white wheat flour.

* Statistical significance, $P<0.05$.

† Subjects are healthy individuals, if not, further details are given.

‡ Unless otherwise stated, total dietary fibre content corresponds to $\mathrm{g} / 100 \mathrm{~g}$ of bread.

$\S$ More information about how AUC, iAUC, iAUCcut and net iAUC are calculated can be found in the article by Brouns et al.(44). Gl and II values are expressed with G as the reference, being assigned the value of 100.

$\|$ Short corresponds to $30 \mathrm{~min}$ proving time, whereas long to $50 \mathrm{~min}$. Desem is the Dutch word for leaven and means a fermented dough.

${ }_{\star \star *}$ Breads $(50 \mathrm{~g}$ of $\mathrm{AC}$ ) were consumed as part of a breakfast. 
retrograded starch, RS3, which is not susceptible to enzymatic digestion. Unlike the above studies, no difference was detected in terms of postprandial glycaemia and GI values between an ordinary white bread and a precooked frozen white bread, which differed only in the baking procedure ${ }^{(37)}$.

Burton \& Lightowler ${ }^{(42)}$ studied the effect of storage and preparation conditions on postprandial glucose response to white bread. In this randomised cross-over design trial, ten healthy volunteers participated. In both homemade and commercial white breads, freezing and defrosting, toasting from fresh and toasting following freezing and defrosting resulted in significantly lower glucose response. The effect of adding extruded chickpea flour to white bread on postprandial glucose and insulin response in healthy subjects was examined by Johnson et al. ${ }^{(43)}$, but results did not show any favourable metabolic effects compared with native chickpea flour.

\section{Discussion}

The present review aimed to give a run down on the human trials that have focused on the effect of bread making technology on postprandial glucose response to bread. Studies examining the postprandial glucose and insulin response after the ingestion of breads differing in the leavening technique used, proofing time or baking process applied are included in this article, aiming to designate effective strategies for the modulation of bread's GI. It is noteworthy that there is an ongoing debate about how reliable the measurements of GI values, and II values, are. Consequently, some variation in measurements on the GI of breads could be attributed to that factor.

Sourdough fermentation of either white or whole meal breads has consistently been shown to attenuate the $\mathrm{GI}^{(7,13,15,32-33)}$. Postprandial glucose response following the consumption of breads leavened with sourdough lactobacilli also results in lower iAUC values, compared with reference foods ${ }^{(14,16,21-23)}$. Wholemeal wheat and white wheat sourdough breads do not exhibit differences in glucose response, as the presence of insoluble fibre does not seem to influence glycaemic potential of breads ${ }^{(21)}$. According to the accumulated data, the lowest GI value has been demonstrated by De Angelis et al. ${ }^{(13)}$, and is a wheat sourdough bread enriched with dietary oat and rye fibre manufactured in industrial plant. Sprouting treatment of whole-grain wheat, which is known to increase the content and availability of vitamins, minerals, antioxidants and phytochemicals, in combination with sourdough fermentation led to the production of breads which exerted lower AUC for glycaemia in patients with $\mathrm{T}_{2} \mathrm{D}^{(22)}$ and overweight or obese subjects $^{(23)}$. The amount of sourdough used for the manufacture of gluten-free breads influences GI value, with moderate amounts (15 and $22.5 \mathrm{~g}$ of sourdough) to exhibit the highest drops in GI, whereas higher amount of sourdough resulted in the lowest drop in GI possibly due to a decrease in viscosity ${ }^{(32)}$. However these results remain to be confirmed in non-gluten-free breads as well. Different starters also lead to different concentration of lactic and acetic acids, thus different GI values. A lower ratio of lactic: acetic acid has been proposed to be associated with greater GI reduction in gluten-free breads ${ }^{(33)}$.
The evidence regarding the effect of sourdough breads consumption on postprandial insulin response is less clear. Among the reviewed studies, II of a sourdough rye bread was evaluated in the study by Goletzke et $a l^{(7)}$ and found 70 (with glucose as reference), whereas Rizkalla et al. ${ }^{(39)}$ reported II 59 for a white bread leavened with sourdough. Lower postprandial insulin response to breads fermented with sourdough, compared with reference foods, has been reported in some studies ${ }^{(16,19-20,23)}$, whereas there are also studies showing no difference ${ }^{(14,22)}$. The consumption of breads prepared with endosperm rye flour has been associated with improved postprandial insulin profile without affecting glucose response ${ }^{(18)}$. In accordance to this observation, rye sourdough breads do not consistently result in attenuated glucose response, as there are data proving beneficial impact $^{(7)}$ as well as data demonstrating no difference in postprandial glycaemia ${ }^{(19,20)}$

The fermentation of wheat and rye flours by lactic acid bacteria results to the formation of organic acids, especially lactic acid, which are mainly responsible for the acute metabolic benefits. Among the mechanisms reported in the literature slower gastric emptying rate ${ }^{(24,26)}$, lower rate of starch digestibility ${ }^{(15)}$, formation of $\mathrm{RS}^{(15,21)}$, interactions between starch and proteins ${ }^{(27)}$, firmer structure especially in rye breads ${ }^{(19)}$, release of amino acids, peptides and free phenolic compounds in the gut ${ }^{(30-31)}$, and proteolysis ${ }^{(16)}$ are included. Available data do not allow drawing any safe conclusions. Fermenting bread with sourdough lactobacilli instead of baker's yeast is potentially an effective strategy for lowering the GI and possibly insulin response of bread products. Besides the beneficial effects of sourdough biotechnology on sensory, structural and shelf life properties, a large body of evidence shows its potential to improve nutritional quality by increasing levels of vitamins and bioactive compounds and improving mineral bioavailability $^{(44)}$.

The manipulation of processing procedures can result in physical structure differences and in turn starch digestibility variation of breads with the same macronutrient composition. Steamed compared with baked white bread has been shown to exert low GI regardless the bread recipe used ${ }^{(5)}$. When ascertaining whether macronutrient composition or processing parameters has a greater impact on postprandial glucose response, the latter was found to play a more pivotal role ${ }^{(5)}$. Similarly the application of pumpernickel bread baking conditions, which are known to hinder the swelling of starch granules thus starch gelatinisation, also lowers postprandial glucose response to bread ${ }^{(35)}$. Reducing bread volume by modifying proofing conditions resulted in significant decrease in bread's GI in the study by Burton \& Lightowler ${ }^{(36)}$. Dense and compact bread structure possibly results in limited accessibility of amylase to gelatinised starch granules. When no resting period is applied (preventing the dough to rise at its maximum capacity) or the gluten content of flour is low, the high porous bread structure cannot be created, which in turn significantly affects glucose response ${ }^{(37)}$. The increase of proofing time from 30 to $50 \mathrm{~min}$ did not significantly alter the GI of bread in another study ${ }^{(38)}$. Another way of achieving a high RS content, thus low GI of baked products, is by applying multiple heating-cooling cycles, which promote the retrogradation of starch. Partial-baking freezing technology combined with either the addition of $10 \%$ dietary fibre ${ }^{(40)}$ or sourdough in low amount $(3 \%)^{(41)}$ has resulted in the design of low 
GI breads. The application of simple household methods such as freezing and toasting of white bread has also shown to favourably alter glucose response to white bread $^{(42)}$

As far as postprandial insulin response is concerned, data has shown lower II for a dense bread (Candeal-flour bread) compared with reference food (glucose) ${ }^{(37)}$, lower iAUC after the ingestion of a pumpernickel rye bread compared with white bread ${ }^{(35)}$ and lower II to a traditional white baguette ${ }^{(39)}$, which artisanal method of preparation did not allow the dough to rise properly. The above initial data suggest that there can be an effect of physical structure of bread on postprandial insulin response, resulting from the retarded glucose release and absorption. However, more randomised controlled trials are needed to understand the potential links of bread microstructure and postprandial insulin response.

\section{Conclusions}

Although bread is daily consumed in almost every part of the world and could be one of the main determinants of dietary glycaemic load, there is great variation in postprandial glucose and insulin responses, coming from baking technology differences. Currently, baked goods are mainly produced by highly industrialised processes, and the traditional long-time fermentation of the dough has been replaced by the use of baker's yeast leavening agents. Use of sourdough fermentation is a challenging technology for attenuating the GI of bread. It constitutes not only a tool to exploit the potential of wheat, rye and whole-grain flours, but also an alternative and effective way to decrease glucose and insulin response to bread, whereas the mechanism behind these pronounced effects are still to be fully elucidated. The alteration of other processing conditions could also constitute an innovative route to beneficially alter the postprandial glucose response to bread products, but more research is required in order to examine the potential effects of this strategy on postprandial insulin response as well. Continuous research for novel baking processes that promote consumers health, through lowering postprandial responses, emphasizes the importance of considering alternative baking technologies for bread production.

\section{Acknowledgements}

N. S. S. was awarded a fellowship by the Greek State Scholarship Foundation.

The authors' contributions are as follows: all authors contributed to the design of the article. N. S. S. contributed to the conception of the literature search strategy, undertook the literature search and wrote the manuscript; A. E. Y. contributed to the conception of the literature search strategy and critically evaluated the document at all stages. V. T. K. had the responsibility for producing the last version of the article.

The authors declare that there are no conflicts of interest

\section{References}

1. Lafiandra D, Riccardi G \& Shewry PR (2014) Improving cereal grain carbohydrates for diet and health. J Cereal Sci 59, 312-326.

2. International Organization for Standardization (2010) Food Products - Determination of the Glycaemic Index (GI) and
Recommendation for Food Classification. ISO 26642. Switzerland: ISO

3. Jenkins DJ, Wolever TM, Taylor RH, et al. (1981) Glycemic index of foods: a physiological basis for carbohydrate exchange. Am J Clin Nutr 34, 362-366.

4. Jenkins DJ, Kendall CW, Augustin LS, et al. (2002) Glycemic index: overview of implications in health and disease. $\mathrm{Am} \mathrm{J}$ Clin Nutr 76, 266S-73S.

5. Lau E, Soong YY, Zhou W, et al. (2015) Can bread processing conditions alter glycaemic response? Food Chem 173, 250-256.

6. Fardet A, Leenhardt F, Lioger D, et al. (2006) Parameters controlling the glycaemic response to breads. Nutr Res Rev $\mathbf{1 9}$, $18-25$.

7. Goletzke J, Atkinson FS, Ek KL, et al. (2016) Glycaemic and insulin index of four common German breads. Eur J Clin Nutr 70, 808-11.

8. Scazzina F, Siebenhandl-Ehn S \& Pellegrini N (2013) The effect of dietary fibre on reducing the glycaemic index of bread. Br J Nutr 109, 1163-74.

9. Stamataki NS, Yanni AE \& Karathanos VT (2016) Non-cereal ingredients for the attenuation of glycaemic response to bread: a review of the clinical evidence. Food Funct 7, 2926-36.

10. Arendt EK, Ryan LA \& Dal Bello F (2007) Impact of sourdough on the texture of bread. Food Microbiol 24, 165-174.

11. De Vuyst L \& Neysens P (2005) The sourdough microflora: biodiversity and metabolic interactions. Trends Food Sci Tech 16, 43-56.

12. Liljeberg HG, Lönner CH \& Björck IM (1995) Sourdough fermentation or addition of organic acids or corresponding salts to bread improves nutritional properties of starch in healthy humans. J Nutr 125, 1503-11.

13. De Angelis M, Damiano N, Rizzello CG, et al. (2009) Sourdough fermentation as a tool for the manufacture of low-glycemic index white wheat bread enriched in dietary fibre. Eur Food Res Technol 229, 593-601.

14. Najjar AM, Parsons PM, Duncan AM, et al. (2009) The acute impact of ingestion of breads of varying composition on blood glucose, insulin and incretins following first and second meals. Br J Nutr 101, 391-398.

15. De Angelis M, Rizzello CG, Alfonsi G, et al. (2007) Use of sourdough lactobacilli and oat fibre to decrease the glycaemic index of white wheat bread. Br J Nutr 98, 1196-205.

16. Lappi J, Selinheimo E, Schwab U, et al. (2010) Sourdough fermentation of wholemeal wheat bread increases solubility of arabinoxylan and protein and decreases postprandial glucose and insulin responses. J Cereal Sci 51, 152-158.

17. Maioli M, Pes GM, Sanna M, et al. (2008) Sourdough-leavened bread improves postprandial glucose and insulin plasma levels in subjects with impaired glucose tolerance. Acta Diabetol 45, 91-96.

18. Rosén LA, Silva LO, Andersson UK, et al. (2009) Endosperm and whole grain rye breads are characterized by low postprandial insulin response and a beneficial blood glucose profile. Nutr J 8, 42.

19. Juntunen KS, Laaksonen DE, Autio K, et al. (2003) Structural differences between rye and wheat breads but not total fiber content may explain the lower postprandial insulin response to rye bread. Am J Clin Nutr 78, 957-964.

20. Bondia-Pons I, Nordlund E, Mattila I, et al. (2011) Postprandial differences in the plasma metabolome of healthy Finnish subjects after intake of a sourdough fermented endosperm rye bread versus white wheat bread. Nutr J 10, 116.

21. Scazzina F, Del Rio D, Pellegrini N, et al. (2009) Sourdough bread: starch digestibility and postprandial glycemic response. J Cereal Sci 49, 419-421. 
22. Tucker AJ, Vandermey JS, Robinson LE, et al. (2014) Effects of breads of varying carbohydrate quality on postprandial glycaemic, incretin and lipidaemic response after first and second meals in adults with diet-controlled type 2 diabetes. J Funct Foods 6, 116-125.

23. Mofidi A, Ferraro ZM, Stewart KA, et al. (2012) The acute impact of ingestion of sourdough and whole-grain breads on blood glucose, insulin, and incretins in overweight and obese men. J Nutr Metab 2012, 184710.

24. Liljeberg HG \& Björck IM (1996) Delayed gastric emptying rate as a potential mechanism for lowered glycemia after eating sourdough bread: studies in humans and rats using test products with added organic acids or an organic salt. Am J Clin Nutr 64, 886-93.

25. Darwiche G, Ostman EM, Liljeberg HG, et al. (2001) Measurements of the gastric emptying rate by use of ultrasonography: studies in humans using bread with added sodium propionate. Am J Clin Nutr 74, 254-8.

26. Liljeberg H, Åkerberg A \& Björck I (1996) Resistant starch formation in bread as influenced by choice of ingredients or baking conditions. Food Chem 56, 389-394.

27. Ostman EM, Nilsson M, Liljeberg-Elmstahl HG, et al. (2002) The effect of lactic acid on blood glucose and insulin responses to cereal products: mechanistic studies in healthy subjects and in vitro. J Cereal Sci 36, 339-346.

28. Gänzle MG, Loponen J \& Gobbetti M (2008) Proteolysis in sourdough fermentations: mechanisms and potential for improved bread quality. Trends Food Sci Technol 19, 513-521.

29. Gobbetti M, Rizzello CG, Di Cagno R, et al. (2014) How the sourdough may affect the functional features of leavened baked goods. Food Microbiol 37, 30-40.

30. Katina K, Laitila A, Juvonen R, et al. (2007) Bran fermentation as a means to enhance technological properties and bioactivity of rye. Food Microbiol 24, 175-186.

31. Katina K, Liukkonen KH, Kaukovirta-Norja A, et al. (2007) Fermentation-induced changes in the nutritional value of native or germinated rye. J Cereal Sci $\mathbf{4 6}, 348-355$.

32. Novotni D, Cukelj N, Smerdel B, et al. (2012) Glycemic index and firming kinetics of partially baked frozen gluten-free bread with sourdough. J Cereal Sci 55, 120-125.
33. Novotni D, Curić D, Bituh M, et al. (2011) Glycemic index and phenolics of partially-baked frozen bread with sourdough. Int J Food Sci Nutr 62, 26-33.

34. Jenkins DJ, Wolever TM, Jenkins AL, et al. (1986) Low glycemic response to traditionally processed wheat and rye products: bulgur and pumpernickel bread. Am J Clin Nutr $\mathbf{4 3}$, 516-520.

35. Breen C, Ryan M, Gibney MJ, et al. (2013) Glycemic, insulinemic, and appetite responses of patients with type 2 diabetes to commonly consumed breads. Diabetes Educ 39, 376-386.

36. Burton P \& Lightowler HJ (2006) Influence of bread volume on glycaemic response and satiety. Br J Nutr 96, 877-882.

37. Gonzalez-Anton C, Rico MC, Sanchez-Rodriguez E, et al. (2015) Glycemic responses, appetite ratings and gastrointestinal hormone responses of most common breads consumed in Spain. A randomized control trial in healthy humans. Nutrients 7, 4033-4053.

38. Fredensborg MH, Perry T, Mann J, et al. (2010) Rising methods and leavening agents used in the production of bread do not impact the glycaemic response. Asia Pac J Clin Nutr 19, 188-94.

39. Rizkalla SW, Laromiguiere M, Champ M, et al. (2007) Effect of baking process on postprandial metabolic consequences: randomized trials in normal and type 2 diabetic subjects. Eur J Clin Nutr 61, 175-183.

40. Borczak B, Sikora E, Sikora M, et al. (2012) Glycaemic response to frozen stored wheat rolls enriched with inulin and oat fibre. J Cereal Sci 56, 576-580.

41. Borczak B, Sikora E, Sikora M, et al. (2011) The impact of sourdough addition to frozen stored wheat-flour rolls on glycemic response in human volunteers. Starch/Stärke $\mathbf{6 3}$, 801-807.

42. Burton P \& Lightowler HJ (2008) The impact of freezing and toasting on the glycaemic response of white bread. Eur J Clin Nutr 62, 594-599.

43. Johnson SK, Thomas SJ \& Hall RS (2005) Palatability and glucose, insulin and satiety responses of chickpea flour and extruded chickpea flour bread eaten as part of a breakfast. Eur J Clin Nutr 59, 169-176.

44. Brouns F, Bjorck I, Fryan KN, et al. (2005) Glycaemic index methodology. Nutr Res Rev 18, 145-71. 